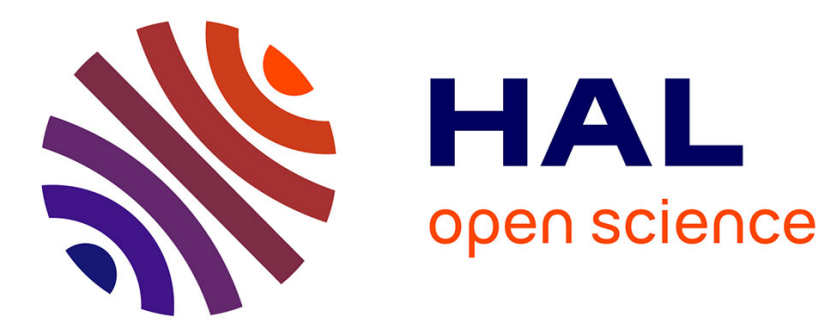

\title{
3D-Aggregate Quantitative Imaging: Experimental Results and Polarization Effects
}

\author{
Christelle Eyraud, Jean-Michel Geffrin, Amelie Litman
}

\section{To cite this version:}

Christelle Eyraud, Jean-Michel Geffrin, Amelie Litman. 3D-Aggregate Quantitative Imaging: Experimental Results and Polarization Effects. IEEE Transactions on Antennas and Propagation, 2011, 59 (4), pp.1237 - 1244. 10.1109/TAP.2011.2109353 . hal-01910338

\section{HAL Id: hal-01910338 \\ https://hal.science/hal-01910338}

Submitted on 31 Oct 2018

HAL is a multi-disciplinary open access archive for the deposit and dissemination of scientific research documents, whether they are published or not. The documents may come from teaching and research institutions in France or abroad, or from public or private research centers.
L'archive ouverte pluridisciplinaire $\mathbf{H A L}$, est destinée au dépôt et à la diffusion de documents scientifiques de niveau recherche, publiés ou non, émanant des établissements d'enseignement et de recherche français ou étrangers, des laboratoires publics ou privés. 


\title{
3D-Aggregate Quantitative Imaging: Experimental Results and Polarization Effects
}

\author{
Christelle Eyraud, Jean-Michel Geffrin, and Amélie Litman, Member, IEEE
}

\begin{abstract}
We present reconstructions of an aggregate of small spheres from experimental scattered fields using a working frequency of $18 \mathrm{GHz}$. This target presents at the same time a complex 3D shape and a low-contrast permittivity. Concerted experimental and numerical efforts have enabled to obtain accurate reconstructions. In particular, we took into account the real random noise via a Bayesian framework. Reconstructions have been realized with scattered fields measured in different polarization cases: the results are compared and discussed.
\end{abstract}

Index Terms-Aggregate, Bayesian formulation, microwave analogy, microwave imaging, 3D inverse scattering problem.

\section{INTRODUCTION}

$\mathbf{E}$ LECTROMAGNETIC wave probing is a useful tool to obtain information from an object in a non destructive way. Indeed, the physical features of an unknown target (position, shape, size, complex permittivity) can be retrieved from its scattered field thanks to the resolution of an inverse problem. This implies accurate measurements of the scattered field associated with an efficient inversion algorithm.

Nowadays, as several inversion procedures have been extended to 3D configurations (see [1]-[3] for example) and as scattered fields of 3D-objects can be precisely measured [4], [5], the characterization of complex shaped 3D-targets can thus be realized. In particular, different inversion algorithms were recently tested against the same experimental dataset $(3 D$ Fresnel database) measured with the Institut Fresnel setup and the results were published in a special section of the Inverse Problems review [6].

In the present work, we are interested in a specific target which presents a particularly complex geometry including small details: an aggregate of spheres. To our knowledge, it is the first time that a target with such a complex geometry has been reconstructed with such a high frequency $(18 \mathrm{GHz})$. To give an example, in the special section of the $3 D$ Fresnel database [5], [6], the highest frequency was $8 \mathrm{GHz}$. To reach such a goal, we took profit of a joint work between the inversion developments and the experimental ones. In particular, (i) we have optimized the quality of the data (knowledge and correction of the experimental errors), (ii) their quantity (number of measurements) and

Manuscript received December 04, 2009; revised March 29, 2010; accepted November 10, 2010. Date of publication February 04, 2011; date of current version April 06, 2011.

The authors are with the Institut Fresnel UMR CNRS 6133, Université Paul Cézanne Aix-Marseille III, Ecole Centrale de Marseille, Université de Provence Aix-Marseille I, Marseille France (e-mail: christelle.eyraud@fresnel.fr).

Color versions of one or more of the figures in this paper are available online at http://ieeexplore.ieee.org.

Digital Object Identifier 10.1109/TAP.2011.2109353

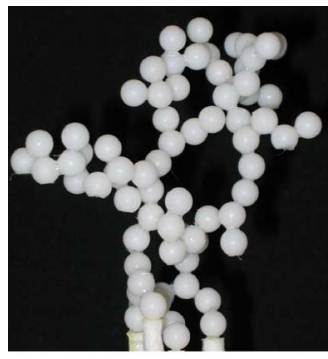

(a)

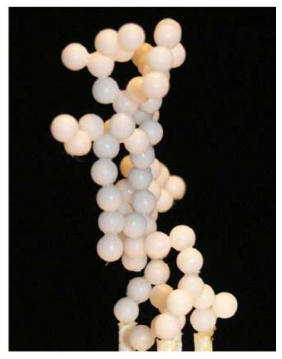

(b)
Fig. 1. Views from different directions of the aggregate, composed of 74 spheres of $2.5 \mathrm{~mm}$ radius. (a) $\phi=90^{\circ}-\theta=0^{\circ}$. (b) $\phi=90^{\circ}-\theta=270^{\circ}$.

(iii) we have based our inversion procedure on a physical description of the problem using a Bayesian approach and a positivity constraint.

The scattered field of this target has been measured in different polarization cases. Indeed, polarization of the illuminating wave and the choice of the polarization of the receiving antenna are two important parameters as geophysical studies or radar studies point it out ([7], [8] for example). In inversion procedures, the different polarization cases are very often considered to contain the same quantity of information [6], [9], [10]. Here, we present reconstructions with different polarization cases and we discuss the interest and relevance of each case either taking each one separately or using various combinations.

This paper is organized as follows. In Section II, the aggregate and its specific characteristics are presented. Sections III and IV explain the experimental study and the inversion procedure, each one being influenced by the other. The results of the reconstructions are shown in Section $\mathrm{V}$ with a discussion on the polarization inference on the reconstructions. Finally, some concluding remarks are provided in Section VI.

\section{The AgGregate}

The studied target is an aggregate of spheres (Fig. 1), composed by 74 dielectric spheres of $2.5 \mathrm{~mm}$ radius. It has a fractal dimension of 1.7 [11]. The spheres are made of polyacetal and the relative permittivity of this material was measured with the commercial kit EpsiMu [12], [13]. The relative permittivity value was then refined comparing diffraction measurements of a single $50 \mathrm{~mm}$ diameter sphere made of the same material and Mie computations [14], [15] with a minimization process. The final value was found to be purely real and equal to $2.85 \pm 0.01$. More details concerning this object and accurate comparisons between simulated and experimental scattered fields can be 

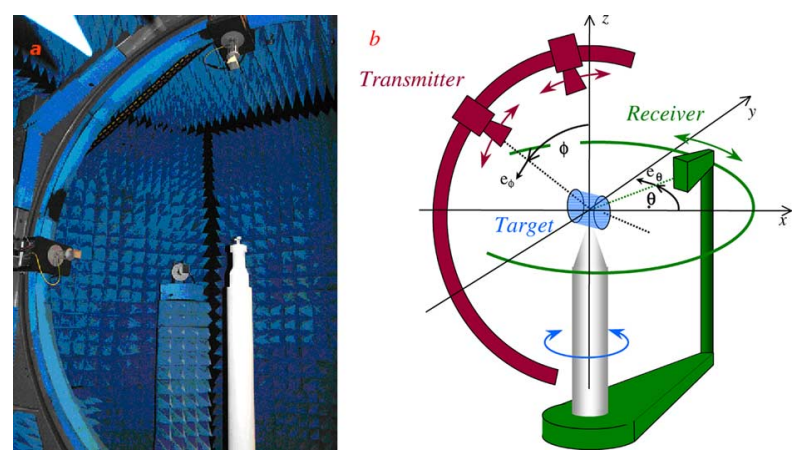

Fig. 2. Measurement setup: (a) picture and (b) sketch.

found in [16] and on this website: http://www.fresnel.fr/3Ddirect/database.php.

This aggregate presents several singular characteristics. Its geometry is entirely non-symmetrical (no symmetry axis or plane). This implies that this target necessitates measurements for several incident and receiver directions to provide the possibility of a good characterization. It is a depolarizing target [17] and thus that measurements of different polarization cases are interesting. These measurements can be realized with our experimental setup almost all around the $2 \mathrm{~m}$ radius sphere enclosing the object [5].

This target has a very low density, i.e., the volume of the material represents less than $3 \%$ of the volume of the minimum sphere including the target, so it is globally a low-contrasted object. This involves that high accuracy on the measured scattered fields is definitely needed. Taking profit of our previous works [4], we are able to eliminate the systematic errors, to correct for the drift errors [18] and to estimate the remnant noise (random noise) on each measured point [19].

This object includes small details compared to its size, i.e., each sphere diameter represents $1 / 20$ of the diameter of the minimum sphere enclosing the target. As these spheres have small dimensions, we chose to realize the inversion process using scattered fields measured at a frequency such that the individual sphere has a diameter of about $\lambda / 3(18 \mathrm{GHz})$ to be able to retrieve such a fine geometry. On the other hand, the perturbations disturbing the scattered field are becoming more important and all the geometrical imprecisions are more sensitive when the frequency increases. In particular, the random noise increases, thus it is specially important to take this noise into account in the inversion process.

\section{EXPERIMENTAL STUDY}

\section{A. System Overview}

The measurements were performed in the faradized anechoic chamber $(14.5 \mathrm{~m} \times 6.5 \mathrm{~m} \times 6.5 \mathrm{~m})$ of the C.C.R.M. (Centre Commun de Ressources en Microondes) in Marseille (Fig. 2), already described in various papers [10], [20]. The equipment is based on a Vector Network Analyzer (HP 8510 B), used in a multiple sources configuration with two synthesizers and two external mixers.

This setup is represented on Fig. 2(a). The transmitting antenna can be moved along the vertical arch in a vertical plane $(\phi$ angle). The target - placed at the top of a cylindrical expanded polystyrene mast at the position $(0,0,0)$ - can rotate on itself around the vertical $z$-axis ( $\theta$ angle) which allows to increase the number of incident directions. $\left(e_{\theta}, e_{\phi}\right)$ are the basis vectors of a spherical coordinate system (Fig. 2(b)), where the conventions for the polar and azimuthal angle notations $(\theta, \phi)$ defined in [21]. To reduce the measurement time, two antennas (in orthogonal polarization) are placed in two different positions on the vertical arch and are selected alternatively as sources using a microwave switch, i.e., for each receiver position, the field is measured successively from two different illuminations without mechanical movements. The receiving antenna is positioned on an arm and can rotate in the horizontal plane $x 0 y$ around the target $(\theta$ angle). The distance between the source and the object (resp. between the receiver and the object) is $1629 \pm 2 \mathrm{~mm}$ (resp. $1702 \pm 2 \mathrm{~mm}$ ).

The transmitting and the receiving antennas are two high gain pyramidal horns (ARA MWH1826B) - to have a scattered field signal as high as possible - with a working frequency between $18 \mathrm{GHz}$ and $26 \mathrm{GHz}$. The cable lengths and the power levels have been optimized so that the level of the forward scattering signal is just below the mixer saturation. These antennas are linearly polarized and can be rotated on their support to change the polarization direction (along $\mathbf{e}_{\boldsymbol{\theta}}$ or $\mathbf{e}_{\boldsymbol{\phi}}$ ).

\section{B. Choice of the Experimental Parameters}

As this target is a depolarizing one, its scattered field was measured in the four polarization cases: $\phi \phi, \theta \theta, \theta \phi$ and $\phi \theta(\theta \phi$ means that the emitter has the polarization $\mathbf{e}_{\boldsymbol{\theta}}$ and the receiver is sensitive to the polarization $\mathbf{e}_{\phi}$ ).

The angular step of the transmitting antenna movement and of the receiving one were chosen in accordance with the maximum spatial frequency of the scattered field at $18 \mathrm{GHz}$. Indeed, the scattered field $\mathbf{E}_{\mathbf{s}}$ of bounded induced sources presents the property of having a limited spectral bandwidth in a spherical configuration when this field is observed far enough from the target [22]. A minimum spatial sampling angle can thus be deduced for the emitter and for the receiver allowing to measure all available information on the target. Indeed, the fields were measured with the step $\Delta \theta=5^{\circ}$ on 53 equidistant points placed on a circle in the horizontal plane $x 0 y-100^{\circ}$ are excluded due to mechanical impossibilities. The aggregate was illuminated with 117 incident directions. For this, the transmitting antenna was moved with the step $\Delta \phi=10^{\circ}$ in the vertical plane realizing 13 incidences and furthermore, for each of those positions, 9 more incidences were created by rotating the target around the $z$-axis with the step $\Delta \theta=40^{\circ}$. This last step is larger than the maximum sampling criterion $\left(11^{\circ}\right)$, but this choice was made to keep a reasonable measurement time.

Finally, a calibration process, using a single reference target (a metallic sphere), estimated in the $\phi \phi$ case only for sake of simplicity [4], [23], was applied to every scattered field keeping the same coefficient in each polarization case.

\section{INVERSION PROCEDURE}

The experimental scattered fields, measured on the antennas sphere (far away from the target), are allowing to deduce the 
$3 \mathrm{D}$ relative permittivity map in the domain $\Omega$ (box including the target) thanks to an appropriate inversion procedure. A Bayesian formulation was used to balance each data according to its accuracy and to describe all available information. To solve this non-linear inverse problem, an iterative scheme is adopted and this necessitates an accurate forward problem.

\section{A. Forward Problem}

We calculate the scattering of a 3D inhomogeneous structure in the frequency domain using a volume integral formulation [24]. The field inside the imaging domain is computed with a biconjugated gradient stabilized FFT method [25] based on a 1D FFT to improve the calculation speed [26]. The computation complexity is then of $O(N \log N)$ and the memory requirement is $O(N)$. The free space dyadic Green tensor is used.

\section{B. Inversion Algorithm}

We chose to introduce information on the effective random noise which disturbs the scattered field measurements in the inversion procedure using a Bayesian formulation. The experimental noise was characterized on the measured fields (the total and incident fields) as explained in a previous study [19]. The results established that the deduced noise on the scattered field can be described by an additive complex random variable whose real and imaginary parts are normally distributed and with associated standard deviations depending on the total field magnitude, on the incident field magnitude and on the frequency. The noise disturbing the scattered field data, for a given frequency, depends thus strongly on the incident direction and on the receiver position. Indeed, the standard deviations can vary by a factor 10 depending on the considered measured point. The Bayesian formulation allows to introduce this knowledge in the inversion procedure. The determination of the unknown relative permittivity map $\varepsilon_{\mathbf{r}}$ with the knowledge of the scattered field measurement $\mathbf{E}_{\mathbf{s}}^{\mathrm{m}}$ can be described by the density of probability [27]-[29]

$$
p\left(\varepsilon_{\mathbf{r}} \mid \mathbf{E}_{\mathbf{s}}^{\mathbf{m}}\right)=\frac{p\left(\boldsymbol{\varepsilon}_{\mathbf{r}}\right) p\left(\mathbf{E}_{\mathbf{s}}^{\mathbf{m}} \mid \varepsilon_{\mathbf{r}}\right)}{p\left(\mathbf{E}_{\mathbf{s}}^{\mathbf{m}}\right)} .
$$

We chose to introduce no statistical information at all on the relative permittivity map, i.e., $p\left(\varepsilon_{\mathbf{r}}\right)$ was assumed to follow an uniform law. Maximizing the distribution to obtain the unknown $\varepsilon_{\mathbf{r}}$ with the knowledge of the measured scattered field $p\left(\varepsilon_{\mathbf{r}} \mid \mathbf{E}_{\mathbf{s}}^{\mathbf{m}}\right)$, the solution corresponds then to the Maximum Likelihood. Thus, as there is no noise correlation from one measurement point to another due to the measurement protocol, the involved costfunctional to minimize can be written as [30]

$$
\begin{aligned}
F\left(\varepsilon_{\mathbf{r}}\right)=\sum_{s=1}^{N_{s}} \sum_{r=1}^{N_{r}} \frac{\left(R e\left[\mathbf{E}_{\mathbf{s}}^{\mathbf{m}}\left(\mathbf{r}_{s}, \mathbf{r}_{r}\right)-\mathbf{E}_{\mathbf{s}, \mathbf{\varepsilon}_{\mathbf{r}}}\left(\mathbf{r}_{s}, \mathbf{r}_{r}\right)\right]\right)^{2}}{\sigma_{R e}^{2}\left(\mathbf{r}_{s}, \mathbf{r}_{r}\right)} \\
+\frac{\left(\operatorname{Im}\left[\mathbf{E}_{\mathbf{s}}^{\mathbf{m}}\left(\mathbf{r}_{s}, \mathbf{r}_{r}\right)-\mathbf{E}_{\mathbf{s}, \varepsilon_{\mathbf{r}}}\left(\mathbf{r}_{s}, \mathbf{r}_{r}\right)\right]\right)^{2}}{\sigma_{I m}^{2}\left(\mathbf{r}_{s}, \mathbf{r}_{r}\right)}
\end{aligned}
$$

where $N_{s}$ (resp. $N_{r}$ ) is the total number of sources (resp. receivers), for the transmitter $\mathbf{r}_{s}$ and the receiver $\mathbf{r}_{r}, \mathbf{E}_{\mathbf{s}, \boldsymbol{\varepsilon}_{\mathbf{r}}}\left(\mathbf{r}_{s}, \mathbf{r}_{r}\right)$ is the simulated scattered field, $\sigma_{R e}\left(\mathbf{r}_{s}, \mathbf{r}_{r}\right)$ and $\sigma_{I m}\left(\mathbf{r}_{s}, \mathbf{r}_{r}\right)$ are the noise standard deviations on the real part and on the imaginary part. With the so-defined cost-functional, for each couple (incident direction, receiver direction), the comparison of the calculated scattered field and of the measured one is balanced with a weighting coefficient - depending on the potential noise disturbing the measurement on this point.

The 3D-complex relative permittivity map of the considered domain $\Omega$ was obtained through an iterative procedure which minimizes the cost-functional $F$ of (2). The minimization is realized with a conjugate gradient algorithm using a positivity constraint and with the standard Polak-Ribiere coefficients [31], [32]. More details about this algorithm can be found in [30].

\section{Reconstruction Error Quantification}

To give a criterion of the reconstruction quality, we have considered different error functions comparing the reconstructed $3 \mathrm{D}$ relative permittivity maps and the true one (due to the difficulty to extract a significative one). It can be noticed that if there is a difference - even weak - between the expected position of the aggregate (considered in the following criteria calculations) and its real position in the experiments, the influence on the criteria can be dramatically strong. Thus, the first step consists in detecting this possible geometrical shift.

1) Estimation of the Real Target Position: We have determined the real position of the center of mass of the aggregate $\mathbf{d}=\left(x_{d}, y_{d}, z_{d}\right)$ using a $3 \mathrm{D}$ cross correlation between the reconstructed contrast map $\left(\varepsilon_{r}-1\right)$ and the expected one

$$
R_{r, t}(\mathbf{d})=\sum_{\mathbf{r}}\left(\varepsilon_{r}^{\text {true }}(\mathbf{r}+\mathbf{d})-1\right) \overline{\left(\varepsilon_{r}^{r e c o}(\mathbf{d})-1\right)}
$$

where $\varepsilon_{r}^{\text {true }}$ is the true relative permittivity value, $\varepsilon_{r}^{r e c o}$ is the reconstructed value at the end of the inversion process and $\mathbf{r}$ describes the position of the considered cell in the $\Omega$ domain. It can be noticed that the cross correlation has been calculated on the ( $\left.\varepsilon_{r}-1\right)$ maps (and not on the $\varepsilon_{r}$ maps) in order to compare only the voxels where some material can be found.

2) Relative Permittivity Map Error Criteria: Once this operation has been realized, we can evaluate the similarity between the two maps. We have considered here four different criteria (the first and the second are standard ones and we propose also two other ones). The first one (resp. second), is a comparison between the two relative permittivity maps with a relative L2 norm (resp. L1 norm)

$$
C^{L i}=\left\|\frac{\varepsilon_{r}^{\text {true }}-\varepsilon_{r}^{\text {reco }}}{\varepsilon_{r}}\right\|_{L i}, \quad i=1,2 .
$$

The third criterion $C^{c c}$, corresponds to the expression of the cross correlation normalized on the contrast maps - for the best position $\mathbf{d}^{*}$ (previously determined)

$$
C^{c c}=\frac{R_{r, t}\left(\mathbf{d}^{*}\right)}{R_{t, t}(\mathbf{0})} .
$$

Its interest is to provide a value linked to the similarity between the two maps comparing only the voxels containing some material with respect to the true map.

To consider the reconstructed relative permittivity also for the empty voxels, we consider a fourth criterion, $C^{c}$ which corresponds to the correlation between the two maps

$$
C^{c}=\frac{\operatorname{Cov}\left(\varepsilon_{r}^{\text {reco }}, \varepsilon_{r}^{\text {true }}\right)}{\sqrt{\operatorname{Var}\left(\varepsilon_{r}^{\text {reco }}\right) \operatorname{Var}\left(\varepsilon_{r}^{\text {true }}\right)}}
$$


where $\operatorname{Cov}(u, v)$ is the covariance between $u$ and $v$ and $\operatorname{Var}(u)$ the variance of $u$. All those parameters have been estimated for each of the reconstructions presented below.

The more similar the two maps are, (i) the closer to 0 the criteria $C^{L 1}$ and $C^{L 2}$ are, (ii) the closer to 1 the criteria $C^{c c}$ and $C^{c}$ are.

\section{Inversion Parameters}

The initial guess of the relative permittivity map was chosen to be equal to the one of the background medium (more precisely, a small constant value was added to avoid the case where the gradients vanish due to the positive constraint expression). The inversion process was stopped when the cost functional is below $3 \sigma$ ( $\sigma$ being the average of the measured noise standard deviation values). For the following reconstructions, the convergence was reached at (or before) the 3 rd iteration. An investigated domain $\Omega$ of $(75 \times 50 \times 75) \mathrm{mm}^{3}$ centered at $(x=0$, $y=0, z=0) \mathrm{mm}$ was considered with a cubic lattice spacing equal to $1.6 \mathrm{~mm}(\lambda / 10)$.

\section{RESULTS}

The inversions were performed in three cases: (i) with or without any information on the real random noise, (ii) separately for different polarization cases of the measured scattered fields and (iii) for several combined polarization cases of the measured scattered fields. The different reconstructions are presented and discussed in this section. Note that, for every reconstruction, the real position of the object was found with the cross-correlation criterion.

\section{A. Target Position}

The cross correlation criterion $R_{r, t}$ (3) allows to determine the real position that the aggregate had during the experiments. For all the polarization cases, the same position was found: $\mathbf{d}^{*}=(1.3,-1,2.9) \mathrm{mm}$. This positioning error is very small considering the fact that the target has to be placed at the center $(0,0,0) \mathrm{mm}$ of our setup, a $4000 \mathrm{~mm}$ diameter sphere.

\section{B. Single Polarization Cases}

1) $\phi \phi$ Polarization: In this section, we present the reconstruction results obtained using the scattered fields in the $\phi \phi$ polarization case only.

The first reconstruction was realized without any use of the random noise knowledge, i.e., which amounts to taking $\sigma_{R e}\left(\mathbf{r}_{\mathbf{s}}, \mathbf{r}_{\mathbf{r}}\right)=\sigma_{I m}\left(\mathbf{r}_{\mathbf{s}}, \mathbf{r}_{\mathbf{r}}\right)=1$ in (2). This reconstruction is presented with a $3 \mathrm{D}$ visualization in Fig. 4. For this figure (and all the similar figures in the following), the iso-surface threshold value is taken when the relative permittivity is different from the free space one and the angles plotted at the top of each view correspond to the (azimuth, elevation) coordinates of the viewpoint. This figure has to be compared with the one of the expected aggregate (Fig. 3). This inversion allows to reconstruct a 3D structure but the fine geometry of the aggregate is not really well obtained, i.e., the outlines are not distinct. Moreover, the maximum relative permittivity value of the reconstructed map is weak: 1.94 (the true one being 2.85).

Secondly, we took into account the experimental random noise in the inversion process with the formulation explained in
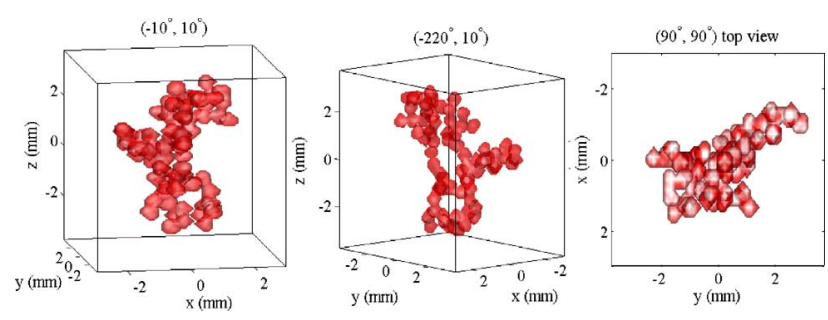

Fig. 3. Real aggregate representation with an iso-surface threshold function.
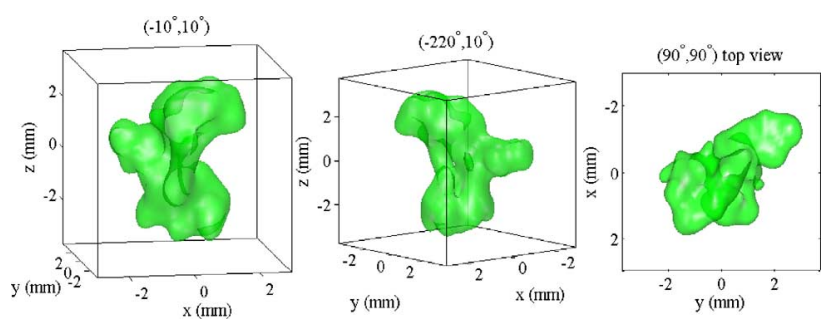

Fig. 4. Reconstruction result at $18 \mathrm{GHz}$ using only the scattered field in the $\phi \phi$ polarization and without taking into account the random noise.
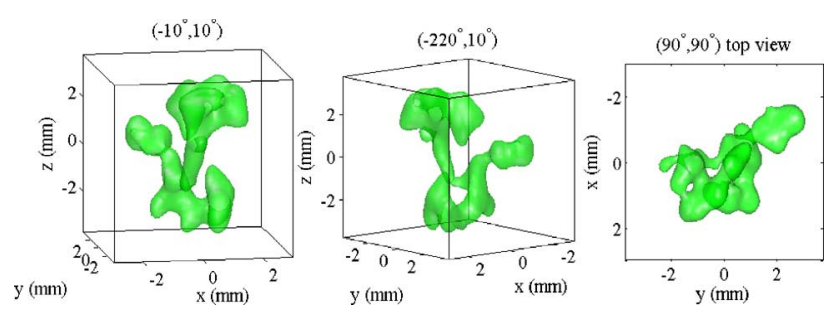

Fig. 5. Reconstruction result at $18 \mathrm{GHz}$ using only the scattered field in the $\phi \phi$ polarization and taking into account the random noise.

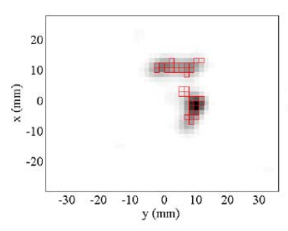

(a)

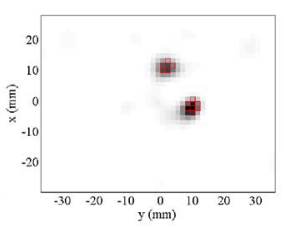

(b)

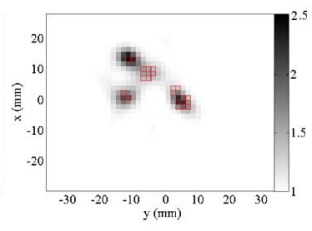

(c)
Fig. 6. Relative permittivity maps reconstructed in the $x 0 y$ plane at $18 \mathrm{GHz}$ and at several altitudes $z$ using scattered fields in the $\phi \phi$ polarization and taking into account the random noise (the colorbar/grayscale is the same for the three plots). (a) $\mathrm{z}=-20 \mathrm{~mm}$. (b) $\mathrm{z}=-15 \mathrm{~mm}$. (c) $\mathrm{z}=+21 \mathrm{~mm}$.

Section IV-B. As it can be seen on Fig. 5, the reconstruction is strongly improved and now the form of the reconstructed object is very close to the real one even if parts of the target (4 spheres) are still missing. The relative permittivity of the target is also higher - the maximum value is now 2.9 - and is similar to the true one (2.85). To study more precisely this result and in particular to visualize the value of the reconstructed relative permittivity, we have plotted the relative permittivity maps at several altitudes $z$ (Fig. 6). On these maps, no interpolation has been done, i.e., the value of each voxel represents the reconstructed value. The voxels with material - considering the true 3D map and the same discretization - are over plotted (overlayed voxels). As it can be seen, the spheres are reconstructed at the proper positions and with a satisfactory relative permittivity value. Each of the associated criteria defined to 
TABLE I

CRITERIA VALUES FOR THE DIFFERENT ReCONSTRUCTION CASES (RAND: WITH EFFECTIVE RANDOM NOISE INFORMATION AND NO: WITHOUT RANDOM NOISE INFORMATION)

\begin{tabular}{|l|c|c|c|c|}
\hline Polarization & $C^{L 2}$ & $C^{L 1}$ & $C^{c c}$ & $C^{c}$ \\
\hline Best case & 0 & 0 & 1 & 1 \\
\hline$\phi \phi^{(\text {no })}$ & 27 & 3087 & 0.200 & 0.535 \\
\hline$\phi \phi^{(\text {rand })}$ & 25 & 1784 & 0.211 & 0.540 \\
\hline$\theta \theta^{\text {(rand })}$ & 23 & 1427 & 0.056 & 0.491 \\
\hline$\phi \phi$ and $\theta \theta^{(\text {rand })}$ & 26 & 1870 & 0.211 & 0.530 \\
\hline$\phi \phi$ and $\phi \theta^{(\text {rand })}$ & 33 & 2531 & 0.258 & 0.443 \\
\hline
\end{tabular}
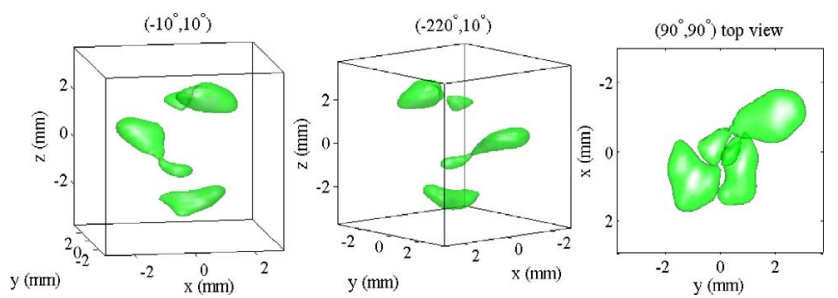

Fig. 7. Reconstruction result at $18 \mathrm{GHz}$ using only the scattered field in the $\theta \theta$ polarization.
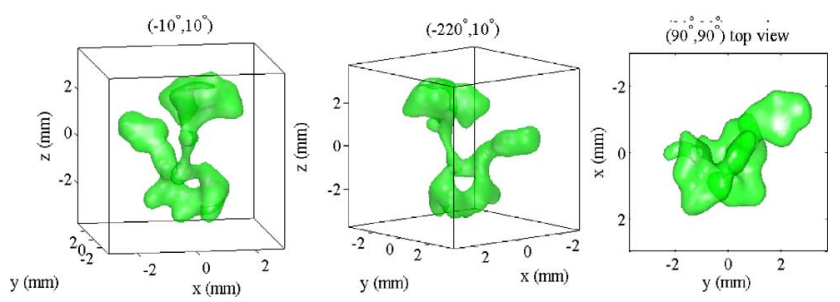

Fig. 8. Reconstruction result at $18 \mathrm{GHz}$ using the scattered field in the $\phi \phi$ and in the $\theta \theta$ polarizations.

quantify the reconstruction error is improved (Table I, line 2). This means that both permittivity estimations of the voxels with and without material are better.

Thus, all the other reconstructions presented in this paper, were obtained taking into account the random noise in the procedure as this knowledge of the real noise does improve the results.

2) $\theta \theta$ Polarization: We now only consider the data measured in the $\theta \theta$ polarization case. As it can be seen in Fig. 7, the horizontal zones are preferentially and almost solely reconstructed. The maximum value of the reconstructed relative permittivity is weak (1.4). For these two reasons, the $C^{c c}$ and $C^{c}$ criteria are low (Table I, line 3). The surprising values of the $C^{L 2}$ and $C^{L 1}$ criteria are due to the fact that these criteria are mainly sensitive to the great number of well reconstructed empty voxels. Physical reasoning is derived in Section V-D to understand the obtained results.

\section{Two Polarization Cases}

In this paragraph, we consider two polarization cases together in the inversion process. As the $\phi \phi$ polarization brings more information than the $\theta \theta$ one, we have kept this polarization and we have tried to add an other polarization to see if we can improve the results.

1) $\phi \phi$ and $\theta \theta$ Polarizations: In this case, the scattered field in the $\phi \phi$ polarization is used in combination with the field measured in the $\theta \theta$ case. As it can be seen on Fig. 8, the recon-
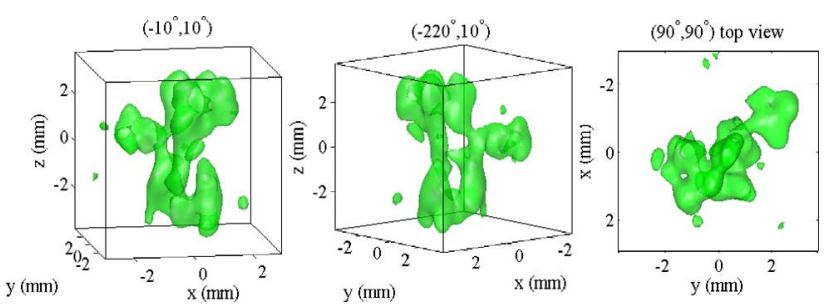

Fig. 9. Reconstruction result at $18 \mathrm{GHz}$ using the scattered field in the $\phi \phi$ and in the $\phi \theta$ polarizations.

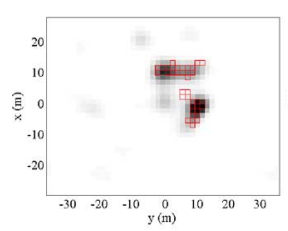

(a)

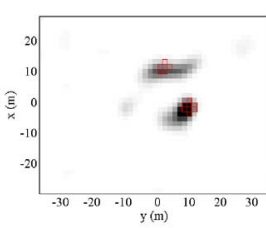

(b)

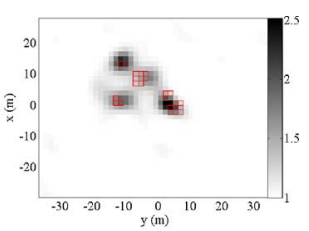

(c)
Fig. 10. Relative permittivity maps reconstructed in the $x 0 y$ plane at $18 \mathrm{GHz}$ and at several altitude $z$ using scattered fields in the $\phi \phi$ and in the $\phi \theta$ polarizations (the colorbar/grayscale is the same for the three plots). (a) $\mathrm{z}=-20 \mathrm{~mm}$. (b) $\mathrm{z}=-15 \mathrm{~mm}$. (c) $\mathrm{z}=+21 \mathrm{~mm}$.

struction is very close to the one obtained considering only the scattered field in the $\phi \phi$ polarization. The cross correlation, the correlation, L2-norm and the L1-norm criteria are also similar to the ones obtained in the $\phi \phi$ case (Table I).

2) $\phi \phi$ and $\phi \theta$ Polarizations: In this case, the scattered field in the $\phi \phi$ polarization is used in combination with the measured field in the $\phi \theta$ case. The reconstruction is presented in the Figs. 9-10. As it can be seen, the voxels including material are more precisely reconstructed (in comparison with the $\phi \phi$ polarization case) and the relative permittivity value is also higher in these zones. The cross correlation criterion $C^{c c}$ is also improved compared to the $\phi \phi$ polarization case (Table I, line 5). It can be noticed that some artefacts did appear, but their values are very weak (the relative permittivity values of these voxels being less than 1.2) and they are emphasized by the threshold representation. Nevertheless, their influences are also visible on the $C^{c}$, $C^{L 2}$ and $C^{L 1}$ criteria which are worse in this case.

The combination of the $\theta \phi$ polarization with the $\phi \phi$ one is not presented here because this is not an interesting case as explained in Section V-D.

\section{Discussion of the Polarization Interest}

We propose here two tools to analyze and understand the reconstructions obtained with the various polarizations.

1) First Tool: The first interpretation (Fig. 11) is based on a representation of the different incident field vectors (in black) for all the source positions. To clarify the representation, only the end of these vectors are plotted (with the + marker) - each vector being brought back at the center of the setup. The projection of the scattered field vector which is measured by the receiving antenna is overlaid (in cyan/gray) for all the receiver positions. These vectors are normalized in order to focus on information provided by the projection angle. This representation was realized for the four polarization cases.

2) Second Tool: The second tool concerns the induced currents in the $\Omega$ zone. It therefore takes into account the depolar- 

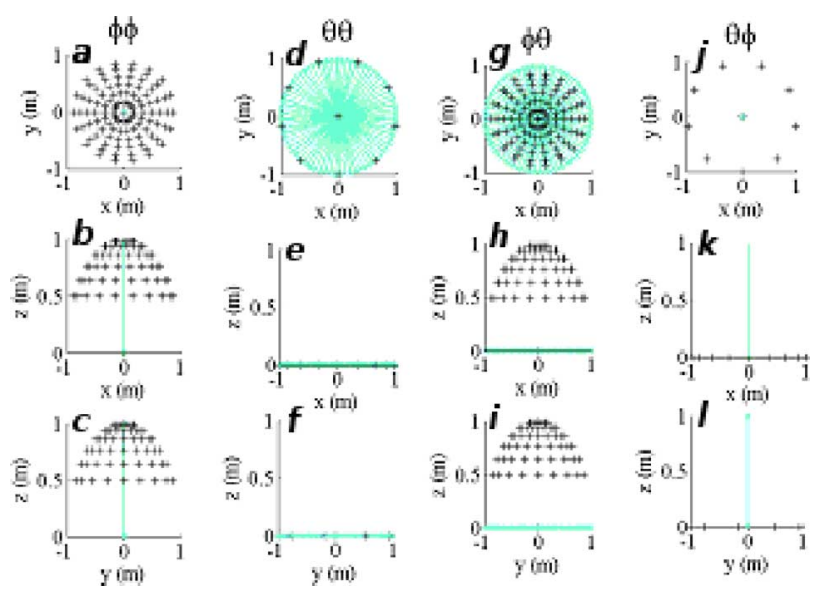

Fig. 11. Representation for $\phi \phi, \theta \theta, \phi \theta$ and $\theta \phi$ polarizations of the incident vectors (black) and of the received scattered vectors (cyan/gray) for all the source and receiver positions - each vector being brought back at the center of the setup - (for the incident vectors, only the end of these vectors are plotted with the marker + ).
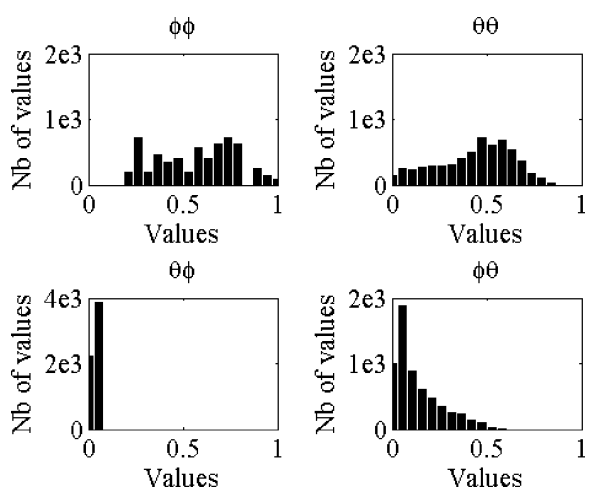

Fig. 12. Histogram of the normalized induced currents projected on the receiver polarization direction for the four polarization cases: $I^{\phi \phi}, I^{\phi \theta}, I^{\theta \theta}$ and $I^{\theta \phi}$ in (7).

izing effects introduced by the target. Indeed, we have numerically calculated the associated direct problem in the configuration of the experiments. We have then observed the values of the projected induced currents on the receiver polarization directions (7) for the four polarization cases $I^{\phi \phi}, I^{\theta \theta}, I^{\phi \theta}, I^{\theta \phi}$ and we have plotted them in terms of histograms (Fig. 12)

$$
I^{S R}\left(\mathbf{r}_{\mathbf{s}}, \mathbf{r}_{\mathbf{r}}\right)=\int_{\Omega}\left|\mathbf{J}^{\mathbf{S}}\left(\mathbf{r}, \mathbf{r}_{\mathbf{s}}\right) \cdot \mathbf{e}^{\mathbf{R}}\left(\mathbf{r}_{\mathbf{r}}\right)\right|^{2} \mathbf{d r}
$$

where $\mathbf{J}^{\mathbf{S}}\left(\mathbf{r}, \mathbf{r}_{\mathbf{S}}\right)$ is the value of the induced current in $\mathbf{r} \in \Omega$ for a source positioned at $\mathbf{r}_{\mathrm{s}}$ with polarization $S$ and $\mathbf{e}^{\mathbf{R}}$ is the unity vector of the measured field for a receiver with polarization $R$.

3) Discussion: We can first observe that the incident polarization along $\phi$ allows to double the number of incident polarization directions (Fig. 11(a)) - compared to the incident polarization along $\theta$ (Fig. 11(d)). Furthermore, the $\phi \phi$ case is also the case containing the highest values of the induced currents (Fig. 12) and thus the signal level is higher and less sensitive to noise [19]. In consequence, the most interesting polarization case is $\phi \phi$. This is in agreement with the quality of the reconstruction obtained using this polarization (Section V-B.I).
Looking to the $\theta \theta$ case (Fig. 11(d)-(f)), the incident field vector is contained in the $x 0 y$ plane for all the source positions. Moreover, all the receivers are only sensitive to the fields contained in the $x 0 y$ plane. Thus, it seems natural that the horizontal geometrical zones of the object are preferentially reconstructed (Section V-B.II). In comparison, it can be noticed that in the $\phi \phi$ case, even if the scattered field is always measured along the $z$ axis, the incident vector has both components in the $x 0 y$ plane and components along the $z$ axis (Fig. 11(a)-(c)).

Considering now the reconstruction with the $\phi \phi$ and the $\theta \theta$ polarizations, we can first note that this combination does not add new incident direction in comparison with the $\phi \phi$ case (Fig. 11(a)-(f)). Looking then to the induced currents (Fig. 12), we can see that, contrarily to our first intuition, the $\theta \theta$ contains only middle range values. These two points can explain why we did not see real improvements in the reconstructions when adding the $\theta \theta$ fields to the $\phi \phi$ ones.

Considering the reconstructions obtained with the $\phi \theta$ and the $\phi \phi$ measurements, it appears that the $\phi \theta$ data are bringing new non redundant - information on the object (Fig. 11(g)-(i)), with respect to the $\phi \phi$ measurement (Fig. 11(a)-(c)). Indeed, in the present case, for the $\phi$ incident wave polarization, the scattered field is measured along the two orthogonal polarizations $\phi$ and $\theta$ - instead of only the $\phi$ one in the $\phi \phi$ case - thus the scattered vector is entirely measured on the receivers for a given illumination. This can explain the improvement of the reconstruction. It can be furthermore noticed that all the incident vector directions in the $\theta \theta$ case are contained in the $\phi \theta$ case as well as all the receiving vector projections.

Finally, looking to the Fig. 11(j)-(l), in the $\theta \phi$ polarization case, (i) the incident and the receiver field vector are always orthogonal, (ii) the target is illuminated with polarization vectors already contained in the $\phi \phi$ polarization and the field is received along the same polarization vector than in the $\phi \phi$ case. Moreover, considering the induced currents, this polarization contains only very few non negligible values (Fig. 12). So, for targets which are not highly depolarizing objects - as the present one, the $\theta \phi$ polarization measurement is not interesting and can be omitted. This can allow to remove a quarter of the measurement time.

To summarize this part, we can remark that these results are not intuitive. Indeed, in our configuration, due to the sources and receivers displacements, the co-polarizations $\phi \phi$ and $\theta \theta$ are not equivalent at all. Moreover it is more useful to add the crosspolarization $\phi \theta$, than the co-polarization $\theta \theta$, to the $\phi \phi$ in this case.

\section{CONCLUSION}

In this paper, our work is based on a good complementarity between the numerical part (positive constraint, Bayesian approach) and the experimental part (number of measurements, calibration, precision of the measurements). This allows to realize faithful reconstructions from measured data of a 3D complex shape target which is, in the present case, an aggregate of 74 spheres. We have also performed similar reconstructions of this object from synthetically generated data - using the same positions of the transmitters/receivers than the ones used in the 
experiments - and as the results are very close to the ones obtained with the measurements, this tends to demonstrate that the concerted experimental and numerical efforts have been properly made.

The evaluation of the reconstructions is not a trivial problem. Four criteria have been considered to evaluate the results. The usually adopted criteria (relative L1 and L2-norms) are not very adapted to evaluate the quality of the reconstruction of the present target. Indeed, their values are particularly dependent on the reconstruction of the empty voxels which are not the most interesting voxels. For these two reasons, we have proposed two other criteria: the cross-correlation $C^{c c}$ and the correlation $C^{c}$. Their variations are more significant and in particular the $C^{c c}$ allows to compare only the voxels within the material.

In this study, inversions have been presented using experimental scattered fields, measured with different polarizations. The results have shown that the choice of the polarizations has a great influence on the reconstruction results and the more the polarizations of the emitter and of the receiver are describing a diversity of directions, the better the results are. With our setup, the $\phi \phi$ polarization is the most interesting case as it contains a larger diversity of polarization directions of the incident wave and because the measured values are relatively high (compared to the other polarization cases). If a second polarization case can be added, the most favorable one seems to be the $\phi \theta$ polarization case even if it corresponds generally to weaker measurement values. It would be interesting, in future work, to corroborate these conclusions with the inversion results obtained with other complex-shape targets.

\section{ACKNOWLEDGMENT}

The authors would like to thank R. Vaillon, B. Lacroix and O. Merchiers from the CETHIL laboratory, Lyon for the design and the realization of the aggregate.

\section{REFERENCES}

[1] S. Y. Semenov, A. E. Bulyshev, A. E. Souvorov, A. G. Nazarov, Y. E. Sizov, R. H. Svenson, V. G. Posukh, A. Pavlovsky, P. N. Repin, and G. P. Tatsis, "Three-dimensional microwave tomography: Experimental imaging of phantoms and biological objects," IEEE Trans. Microw. Theory Tech., vol. 48, pp. 1071-1074, Jun. 2000.

[2] A. Abubakar and P. M. van den Berg, "Iterative forward and inverse algorithms based on domain integral equation for three-dimensional electric and magnetic objects," J. Comput. Phys., vol. 195, pp. 236-262, Mar. 2004.

[3] J. D. Zaeytijd, A. Franchois, and J.-M. Geffrin, "A new value picking regularization strategy-application to the 3-d electromagnetic inverse scattering problem," IEEE Trans. Antennas Propag., vol. 57, pp. 1133-1149, Apr. 2009.

[4] C. Eyraud, J.-M. Geffrin, P. Sabouroux, P. Chaumet, H. Tortel, H. Giovannini, and A. Litman, "Validation of a 3D bistatic microwave scattering measurement setup," Radio Sci., vol. 43, p. RS4018, 2008.

[5] J.-M. Geffrin and P. Sabouroux, "Fresnel database continuation: Experimental setup and improvements for 3D scattering measurements," Inverse Prob., vol. 25, p. 024001, Feb. 2009.

[6] A. Litman and L. Crocco, "Guest editor introduction," Inverse Prob., vol. 26, p. 020201, Feb. 2009.

[7] Y. Wang and J. Saillard, "Characterization of the scattering centers of a radar target with polarization diversity using polynomial rooting," in Proc. IEEE Int. Conf. on Acoustics, Speech, and Signal Processing, Salt Lake City, UT, May 2001, pp. 2893-2896.
[8] A. Freeman and S. L. Durden, "A three-component scattering model for polarimetric sar data," IEEE Trans. Geosci. Remote Sensing, vol. 36, pp. 963-973, 1998.

[9] K. Belkebir and M. Saillard, "Special section on testing inversion algorithms against experimental data: Inhomogeneous targets," Inverse Prob., vol. 21, pp. S1-S3, 2005.

[10] J.-M. Geffrin, P. Sabouroux, and C. Eyraud, "Free space experimental scattering database continuation: Experimental setup and measurement precision," Inverse Prob., vol. 21, pp. S117-S130, Nov. 2005.

[11] O. Merchiers, J.-M. Geffrin, R. Vaillon, P. Sabouroux, and B. Lacroix, "Microwave analog to light scattering measurements on a fully characterized complex aggregate," Appl. Phys. Lett., vol. 94, p. 94:181107, May 2009.

[12] D. Ba and P. Sabouroux, "Epsimu, a toolkit for permittivity and permeability measurement in microwave domain at real time of all materials: Applications to solid and semisolid materials," Microw. Opt. Technol. Lett., vol. 52, pp. 2643-2648, Dec. 2010.

[13] E. Vanzura, J. Baker-Jarvis, J. Grosvenor, and M. Jasenik, "Intercomparison of permittivity measurements using the transmission/reflection method in 7-mm coaxial transmission lines," IEEE Trans. Microw. Theory Tech., vol. 42, pp. 2063-2070, Nov. 1994.

[14] M. Born and E. Wolf, Principles of Optics. Oxford, U.K.: Pergamon Press.

[15] H. Tortel, "Localization and derivation of an optimal sphere for 3d perfectly conducting objects," J. Electromagn. Waves Applicat., vol. 16 pp. 771-791, Jun. 2002.

[16] O. Merchiers, C. Eyraud, J.-M. Geffrin, R. Vaillon, B. Stout, P. Sabouroux, and B. Lacroix, "Microwave measurements of the full amplitude scattering matrix of a complex aggregate: A database for the assessment of light scattering codes," Opt. Express, vol. 18, p. 121191, Jan. 2010.

[17] C. Monzon, "A cross-polarized bistatic calibration device for rcs measurements," IEEE Trans. Antennas Propag., vol. 51, pp. 833-839, 2003.

[18] C. Eyraud, J.-M. Geffrin, A. Litman, P. Sabouroux, and H. Giovannini, "Drift correction for scattering measurements," Appl. Phys. Lett., vol. 89, p. 244104, Dec. 2006.

[19] J.-M. Geffrin, C. Eyraud, A. Litman, and P. Sabouroux, "Optimization of a bistatic microwave scattering measurement setup: From high to low scattering targets," Radio Sci., vol. 44, p. RS003837, Mar. 2009.

[20] P. Sabouroux, J.-M. Geffrin, and C. Eyraud, "An original microwave near-field/ far-field spherical setup: Applications to antennas and scattered field measurements," in Proc. Antenna Measurement Tech. Assoc. Symp., Newport, RI, Nov. 2005, pp. 292-296.

[21] D. Zwillinger, Crc Standard Mathematical Tables and Formulae, 31st ed. London, U.K.: Chapman and Hall/CRC, 2002.

[22] O. M. Bucci and T. Isernia, "Electromagnetic inverse scattering: Retrievable information and measurement strategies," Radio Sci., vol. 32, pp. 2123-2137, 1997.

[23] P. M. van den Berg, M. G. Coté, and R. E. Kleinman, "Blind shape reconstruction from experimental data," IEEE Trans. Antennas Propag., vol. 43, pp. 1389-1396, 1995.

[24] J. A. Kong, "Electromagnetic wave theory," EMW, 2000.

[25] H. A. van der Vorst, Iterative Krylov Methods for Large Linear Systems. Cambridge, U.K.: Cambridge Univ. Press, 2003.

[26] B. E. Barrowes, L. F. Teixeira, and J. A. Kong, "Fast algorithm for matrix-vector multiply of asymmetric multilevel block-Toeplitz matrices in 3-D scattering," Microw. Opt. Technol. Lett., vol. 31, pp. 28-32, 2001.

[27] G. Demoment and J. Idier, "Problèmes inverses : De l'expérimentationà la modélisation: Approche bayésienne pour la résolution des problèmes inverses en imagerie," Observatoire Francais des Techniques Avancées, vol. 22, pp. 59-77, 1999.

[28] A. Baussard, D. Prémel, and Venard, "A Bayesian approach for solving inverse scattering from microwave laboratory-controlled data," Inverse Prob., vol. 17, pp. 1659-1669, 2001.

[29] O. Féron, B. Duchêne, and A. Mohammad-Djafari, "Microwave imaging of inhomogeneous objects made of a finite number of dielectric and conductive materials from experimental data," Inverse Prob., vol. 21, pp. S95-S115, Nov. 2005.

[30] C. Eyraud, A. Litman, A. Hérique, and W. Kofman, "Microwave imaging from experimental data within a Bayesian framework with realistic random noise," Inverse Prob., vol. 26, p. 024005, Feb. 2009.

[31] E. Polak, Computational Methods in Optimization. New York: Academic Press, 1971.

[32] R. E. Kleinman and P. M. van den Berg, "Two-dimensional location and shape reconstruction," Radio Sci., vol. 29, pp. 1157-1169, 1994. 
Christelle Eyraud was born in France in 1980. She received the Ph.D. degree in physics from the Université Aix Marseille I, France, in 2006.

She worked on measurement errors analysis for microwave scattering phenomena. After a postdoctoral position at the laboratory PALMS, Université Rennes 1, France, she worked, as a Postdoctoral Researcher, at the Laboratoire de Planétologie de Grenoble, France, on a 3D inverse algorithm taking into account the measurement uncertainties. In 2008, she joined the Institut Fresnel, Université Aix-Marseille, CNRS, Ecole Centrale Marseille, France, as an Assistant Professor. Her research interests include inverse scattering problems and measurement techniques.

Jean-Michel Geffrin received the Ph.D. degree in physics from the University of Paris XI, France, in 1993.

He worked for ten years as a Research Engineer in France, where he developed specific antennas and experimental setups for measuring targets radiation pattern. In 2002, he joined the Institut Fresnel, Université Aix-Marseille, CNRS, Ecole Centrale Marseille, France, to reinforce the hyperfrequency experimentalist team. He has contributed to the constitution of the second and third databases of scattered fields proposed by the Institut Fresnel to the inverse problem community http://www.fresnel.fr/3Ddatabase/database.php and to the direct database proposed to the (light) scattering community http://www. fresnel.fr/3Ddirect/database.php.
Amélie Litman (M'97) was born in France in 1972. She received the Ph.D degree in applied mathematics from the University of Paris XI, Paris, France, in 1997.

During 1997-1998, she was with the Eindhoven University of Technology, Eindhoven, The Netherlands, working in a postdoctoral position. From 1998 to 2002, she was with Schlumberger, France, where she worked on the development of inversion algorithms for oil prospecting. In October 2002, she joined Institut Fresnel, Université Aix Marseille, CNRS, Ecole Centrale Marseille, France, as an Assistant Professor. Her research interests include forward and inverse scattering techniques. 